2005, she had increasing shortness of breath with intermittent chest pain, which led to rehospitalization for clinical assessment and treatment for heart failure. Echocardiographic study showed LVEDD $62 \mathrm{~mm}$, LVEF 45\%, and grade 3 aortic regurgitation of the ISPB. Blood chemistry studies showed no abnormalities. After successful treatment for heart failure and exclusion of coronary artery disease by coronary angiography, she underwent replacement of the ISPB with a 23-mm Hancock porcine bioprosthesis (Medtronic, Inc, Minneapolis, Minn) on April 25, 2005. The explanted ISPB showed dystrophic changes in all 3 cusps (Figures 1 and 2). The postoperative course was uneventful. The LV function had recovered after 3 months (ejection fraction 55\%, LVEDD $55 \mathrm{~mm}$ ). Clinically, she was in good cardiovascular condition (New York Heart Association functional class II) both at an outpatient visit in August 2005 and at a telephone interview on January 17, 2006.

\section{Comments}

This single case report demonstrates that there is potential in the overall performance of the new-generation pericardial bioprostheses, particularly in relation to structural valve deterioration in selected patients younger than 70 years of age.

We have learned from this single case that pericardial bioprosthesis in general can be implanted in a 50-year-old patient with comorbidities. Infective endocarditis of a pericardial prosthetic valve is a relatively uncommon complication occurring in $0.2 \%$ / patient-year, ${ }^{1}$ and its late occurrence is less common. Acceleration of structural deterioration of the pericardial tissue valve resulted in valve incompetence and compromised the LV function in this patient.

The low-profile ISPB demonstrated excellent hemodynamic performance and had preserved the LV function for more than 22 years. Finally, this case also supports the fact that new-generation pericardial bioprostheses may show the same long-term durability in patients younger than 70 years of age with comorbidities, as was demonstrated in our 17 -year clinical study, ${ }^{1}$ as well as in other publications. $^{2}$

We thank Professor Hans Borst for his contribution and Anne Gale for editorial assistance.

\title{
The disk of bileaflet mechanical mitral valve prosthesis jammed by ruptured papillary muscle
}

\author{
Marcin Fijalkowski, MD, Andrzej Koprowski, MD, PhD, Rafal Pawlaczyk, MD, PhD, Piotr Siondalski, MD, PhD, \\ Rafal Galaska, MD, Jan Rogowski, MD, PhD, and Andrzej Rynkiewicz, MD, PhD, Gdansk, Poland
}

$\mathrm{T}$ he incidence and natural history of papillary muscle rupture occurring after chord-sparing mitral valve replacement for ischemic mitral insufficiency are unknown. A case in which this complication occurred after chord-sparing mitral valve replacement is described.

\section{Clinical Summary}

A 59-year-old man was admitted to our institution with acute heart failure symptoms and chest discomfort lasting for 3 days. The

From the First Department of Cardiology and Department of Cardiosurgery, Medical University of Gdansk, Gdansk, Poland.

Received for publication April 10, 2006; accepted for publication April 20, 2006.

Address for reprints: Marcin Fijalkowski, MD, First Department of Cardiology and Department of Cardiosurgery, Medical University of Gdansk, ul. Debinki 7, 80-952 Gdansk, Poland (E-mail: mfijalkowski@amg.gda.pl).

J Thorac Cardiovasc Surg 2006;132:428-30

$0022-5223 / \$ 32.00$

Copyright $\odot 2006$ by The American Association for Thoracic Surgery doi:10.1016/j.jtcvs.2006.04.018

\section{References}

1. Yankah AC, Schubel J, Buz S, Siniawski H, Hetzer R. Seventeen-year clinical results of 1,037 Mitroflow pericardial heart valve prostheses in the aortic position. J Heart Valve Dis. 2005;14:172-9.

2. Minami K, Boethig D, Mirow N, Kleikamp G, Koertke H, Godehardt E, et al. Mitroflow pericardial valve prosthesis in the aortic position: an analysis of long-term outcome and prognostic factors. J Heart Valve Dis. 2000;9:112-22. electrocardiogram showed sinus tachycardia (115 beats/min) and pathologic Q waves in the II, III, and aVF leads. The serum concentrations of creatine kinase $\mathrm{MB}$ and troponin I were significantly elevated. A chest radiograph revealed pulmonary edema. Two-dimensional transthoracic echocardiography showed akinesia of inferior and posterior walls and severe mitral regurgitation caused by posterior leaflet restriction (vena contracta $7 \mathrm{~mm}$ ). Coronary angiography showed occluded right and circumflex coronary arteries and a critically narrowed left anterior descending coronary artery.

On the basis of these findings, mitral valve surgery and coronary artery bypass grafting were recommended. Treatment with intra-aortic balloon pump support was started, without significant improvement. Emergency cardiosurgery was performed because of the patient's unstable condition. The mitral valve was replaced with a 29-mm Medtronic Advantage prosthesis (Medtronic, Inc, Minneapolis, Minn) placed in antianatomic position with preservation of the posterior leaflet (ring annuloplasty was not successful). Coronary artery revascularization was done in the same operation.

The early postoperative course was uneventful. At the end of the second week of hospitalization, hypotension and pulmonary 

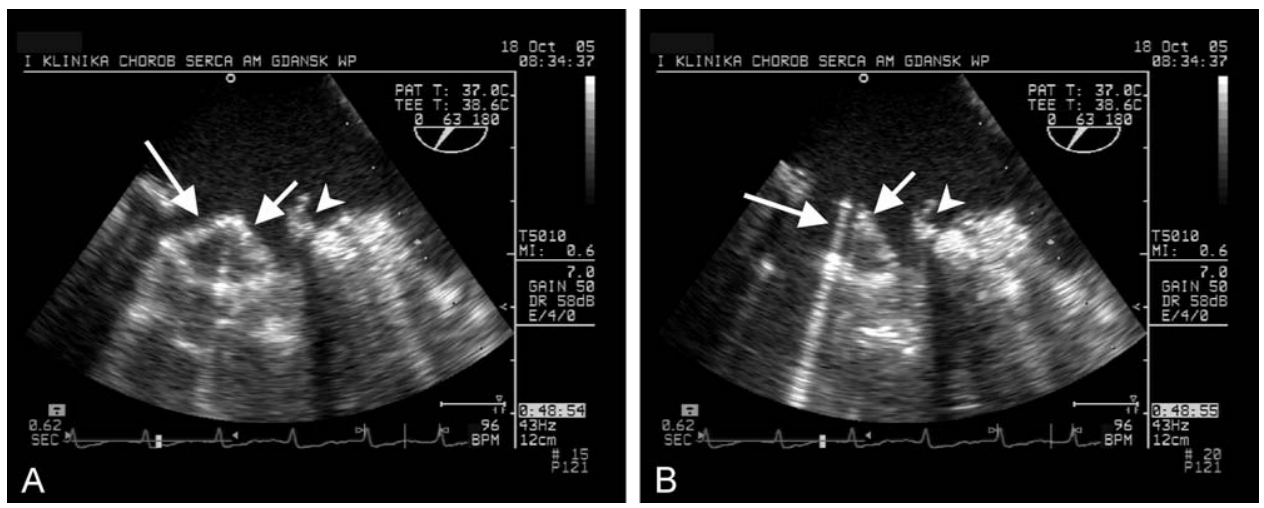

Figure 1. Transesophageal echocardiography shows one normal prosthetic valve disk motion (long arrow) and one immobilized (short arrow) with close additional echo (arrowhead). A, Shows $63^{\circ}$ view in systole. B, Shows $63^{\circ}$ view in diastole.

edema occurred, associated with a decrease in the loudness of prosthetic valve sounds. Transesophageal echocardiography revealed that one of the prosthetic disks did not move during the cardiac cycle and stayed in a semiopen position (Figure 1), leading to severe regurgitation. There was no echocardiographic evidence of prosthetic valve thrombosis, but additional echo was seen close to the entrapped disk (Figure 1). Reoperation revealed that the head of the anteroseptal papillary muscle attached to its chordae tendineae had ruptured and wedged between one of the disks and prosthetic valve ring, resulting in the jamming of the disk in a semiopen position (Figure 2). The mitral valve Medtronic Advantage bileaflet prosthesis was replaced with a 27-mm On-X valve prosthesis (Medical Carbon Research Institute, LLC, Austin, Tex). This time, the posterior leaflet and subvalvular apparatus were surgically removed. The postoperative course was complicated by ischemic stroke.

\section{Discussion}

Papillary muscle rupture is a complication that occurs in a minority of cases of acute myocardial infarction. ${ }^{1}$ The questions raised by this case concern the incidence of postoperative papillary muscle rupture after chord-sparing mitral valve replacement for ischemic mitral regurgitation, as well as the consequences of a flail papillary muscle segment in the left ventricle, especially its interference with the prosthetic valve function. There have been reports of

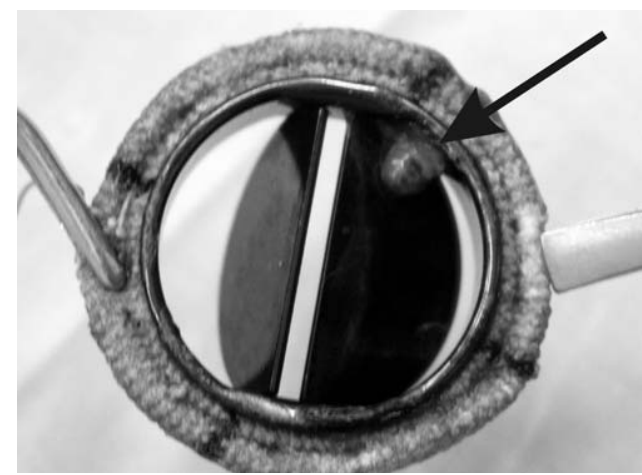

Figure 2. Head of papillary muscle (arrow) wedged between disk and prosthetic valve ring. valve prosthetic disk entrapment by surgically divided chordal remnants, overhanging knots, long suture ends, and atrial catheters. ${ }^{2}$ Spontaneous rupture of papillary muscle with entrapment of the tilting disk of a Medtronic Hall prosthesis has been reported only twice, in both cases causing death. ${ }^{3,4}$ Lemke and colleagues ${ }^{5}$ described a case of spontaneous rupture of papillary muscle after mitral valve replacement, with normal function of mitral prosthesis and floating ruptured papillary muscle prolapsing through the normal aortic valve.

To our knowledge, there are no reports on disk entrapment of the bileaflet mechanical mitral valve prosthesis caused by spontaneous papillary muscle rupture with preserved chordae tendineae. The case reported here is unusual in that the one of the disks of a bileaflet prosthetic valve was jammed in a semiopen position while the other disk function remained normal. It is common knowledge that preservation of the subvalvular structures - the continuity of the mitral annulus, chordae tendineae, and papillary musclesduring mitral valve replacement is important in maximizing ventricular function and maintaining normal ventricular geometry. Mok and associates ${ }^{4}$ described hemorrhagic necrosis of the papillary muscle, possibly related to subendocardial ischemia, as the cause of rupture in 1 case after mitral valve replacement. It is likely in our case that rupture of the head of papillary muscle was due to stress on the ischemic papillary muscle by increased tension related to preservation of the chordae tendineae when the posterior leaflet was oversewn into the base of the prosthetic mitral valve. There is also the possibility that the change in the left ventricular geometry after the operation may have led to increased stress on the chordae tendineae. For those reasons, every effort should be made to avoid excessive tension on the papillary muscle whenever chordal preservation is done, particulary when the papillary muscle could be ischemic. In view of the current widespread use of chord-preserving techniques in ischemic mitral valve replacement, the problem presented in this report is likely to be encountered again in the future.

\section{References}

1. Goldenberg MR, Rozanski LT, Degeratu FT, Berger BC. Papillary muscle rupture after chordal sparing mitral valve replacement. J Heart Valve Dis. 1998;7:590-2. 
2. Williams DB, Pluth JR, Orszulak TA. Extrinsic obstruction of the Bjork-Shiley valve in the mitral position. Ann Thorac Surg. 1981;32: 58-62.

3. Trites PN, Kiser JC, Johnson C, Tycast FJ, Gobel FL. Occlusion of Medtronic Hall mitral valve prosthesis by ruptured papillary muscle and chordae tendineae. J Thorac Cardiovasc Surg. 1984;88:301-2.
4. Mok CK, Cheung DL, Chiu CS, Aung-Khin M. An unusual lethal complication of preservation of chordae tendineae in mitral valve replacement. J Thorac Cardiovasc Surg. 1988;95:534-6.

5. Lemke P, Roth M, Kraus B, Hohe S, Klovekorn WP, Bauer EP. Ruptured papillary muscle after mitral valve replacement with preservation of chordae tendineae. Ann Thorac Surg. 2001;72:1384-6.

\section{Chondromyxoid fibroma of the sternum}

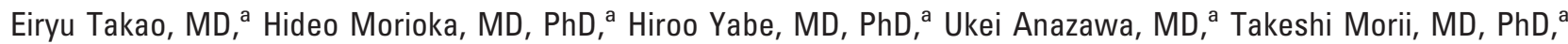

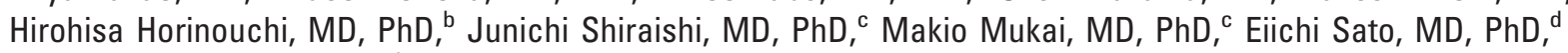
Yoshiki Hamada, MD, PhD, and Yoshiaki Toyama, MD, PhD, ${ }^{\text {a }}$ Tokyo and Yamanashi, Japan

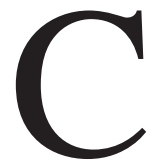

hondromyxoid fibroma (CMF) was described by Jaffe and Lichtenstein ${ }^{1}$ in 1948 and is thought to be a rare benign bone tumor, representing less than $1 \%$ of the total number of primary bone tumors. ${ }^{2}$ It predominantly affects the long bones and rarely occurs in the sternum. In this communication we report a case of CMF of the sternum with a brief review of the literature.

\section{Clinical Summary}

The patient was a 17-year-old man whose chief complaint was pain in the anterior part of the chest. He had occasionally experienced anterior chest pain since about August 2000 and had consulted a local physician because the pain was aggravated by a fall in June 2002. A plain x-ray examination had revealed a bone tumor in the sternum. The patient was first referred to us in July 2002, and he was admitted for surgery in September 2002. During the initial physical examination, a tender swelling that was mildly warm to palpation was found in the middle of the lower sternum. A plain x-ray film showed an eccentrically located multilobular radiolucency with mild calcification in the lower sternum. Computed tomography revealed an osteolytic lesion with discrete calcification in the bone marrow of the sternum (Figure 1, A). The lesion was visualized as an isointense area by T1-weighted mag-

From the Departments of Orthopaedic Surgery and Thoracic Surgery, ${ }^{\mathrm{b}}$ School of Medicine, Keio University, Tokyo, Japan; the Department of Diagnostic Pathology, Keio University Hospital, Tokyo, Japan'; and the Department of Orthopaedic Surgery, University of Yamanashi, Faculty of Medicine, Yamanashi, Japan. ${ }^{\mathrm{d}}$

Received for publication March 15, 2006; accepted for publication April 20, 2006.

Address for reprints: Hideo Morioka, MD, PhD, Department of Orthopaedic Surgery, School of Medicine, Keio University, 35 Shinanomachi, Shinjuku-ku, Tokyo 160-8582, Japan (E-mail: morioka@sc.itc.keio.ac.jp).

J Thorac Cardiovasc Surg 2006;132:430-1

$0022-5223 / \$ 32.00$

Copyright $\odot 2006$ by The American Association for Thoracic Surgery doi:10.1016/j.jtcvs.2006.04.023 netic resonance imaging and as a well-marginated high-intensity area by $\mathrm{T} 2$-weighted magnetic resonance imaging with a slightly lobular pattern (Figure 1, B). On the gadolinium-enhanced T1weighted image the tumor was well enhanced. On the basis of these findings, a malignant cartilaginous tumor of the sternum was suspected, and a biopsy of the lesion was obtained.

Histologic examination of the biopsy specimen revealed a slightly lobular architecture composed of myxoid matrix populated by stellate or elongate cells with eosinophilic cytoplasmic processes. These myxoid areas were interspersed in a background of more cellular regions containing dilated sinusoidal vessels and focal hemorrhage. These findings were compatible with $\mathrm{CMF}$ because there were practically no mitotic figures and the cellular atypia was minimal (Figure 2, $A$ and $B$ ). On the basis of these histologic findings, the following surgical procedure was performed. A part of the cortex was removed from the anterior surface of the sternum. The lesion in the bone marrow was completely curetted, and the remaining lesion was ground off with an air drill. The space after curettage was completely filled with bone allograft fragments. The histologic diagnosis of the surgical specimen was CMF as suspected from the biopsy. The patient's clinical course has been uneventful with no local recurrence thus far, 3 years 6 months postoperatively.

\section{Discussion}

CMF is the least common benign cartilaginous tumor, and only 106 cases were registered in the Japanese Bone Tumor Registry in the 21 years from 1972 to $1993 .^{3}$ It accounts for $0.45 \%$ of all primary bone tumors. The most prevalent site is the metaphysis of long bone, and the affected sites reported have been the tibia in 33 cases $(31 \%)$, the femur in 23 cases $(22 \%)$, and the humerus in 9 cases $(8.5 \%)$. During the above period, 71 cases of sternal primary bone tumors were reported, including 18 cases of osteochondroma, 15 cases of chondrosarcoma, and 14 cases of chondroma. Among these tumors, particular caution is required to differentiate chondrosarcoma from CMF. Because of the presence of pleomorphic cells with hyperchromatic nuclei, the histologic diagnosis of CML may sometimes be difficult and an erroneous diagnosis of chondrosarcoma may result.

To the best of our knowledge, there have been no reports of $\mathrm{CMF}$ 